\title{
Editorial
}

\section{Rückblick im Umbruch}

\section{Zur Geschichte der Psychologie und Psychotherapie in Deutschland}

\author{
Martin Wieser ${ }^{1}$ und Lisa Malich ${ }^{2}$ \\ ${ }^{1}$ Sigmund Freud PrivatUniversität Berlin \\ ${ }^{2}$ Universität zu Lübeck
}

Eine neue Epoche im Verhältnis von Psychologie und Psychotherapie in Deutschland kündigt sich an. Zwanzig Jahre nach dem Inkrafttreten des ersten Psychotherapeutengesetzes verabschiedeten Bundestag und Bundesrat 2019 schließlich das Gesetz zur Reform der Psychotherapeutenausbildung. 2020 trat die neue Approbationsordnung in Kraft. Die Einführung polyvalenter Bachelorstudiengänge folgte auf dem Fuß, kurz darauf die Einrichtung der entsprechenden Masterstudiengänge, die Studierende künftig mit der Approbationsprüfung beenden werden. Die Bewertungen dieses Prozesses für die Zukunft der Psychologie gehen auseinander: Während einerseits auf die Vorteile einer Akademisierung der Psychotherapie hingewiesen wird (Rief, 2020) warnen andere vor einem drohenden Bedeutungsverlust der nicht-klinischen Fächer in der Psychologie (Schönpflug, 2019; Tuschen-Caffier et al., 2020). Ob Gefahr oder Chance: Unbestritten ist die Tatsache, dass die anhaltenden Debatten über die Umstrukturierung des Faches von der immensen Dynamik dieses Reformprozesses zeugen. Es kündigt sich ein tiefgreifender Wandel sowohl der akademischen Psychologie als auch der psychotherapeutischen Profession in Deutschland an.

Wer die Weichen für die Zukunft stellt, lege die Ohren auf die Schienen der Geschichte: In der Retrospektive offenbart sich so mancher Streit von heute als ungewolltes Erbe einer längst vergangenen Zeit, dessen Implikationen und Konsequenzen unter den gegenwärtigen Bedingungen neu zu klären und zu prüfen sind. Nicht nur innerhalb des Faches, sondern auch zwischen den Fakultäten repräsentiert die Frage nach dem Verhältnis von Psychologie und Psychotherapie einen altbekannten Zankapfel in Deutschland. Die Eigentümlichkeiten dieses über Jahrzehnte hindurch gewachsenen Feldes lassen sich nur vor dem Hintergrund seiner historischen Genese begreifen. Diese Überlegungen gaben den Ausgangspunkt für die vorliegende Ausgabe der Psychologischen Rundschau.

\section{Die Psychologie - eine Disziplin im Wachstum}

Um was für eine Disziplin handelt es sich, die nun einen derartigen Transformationsprozess durchläuft? Der Blick auf die Studierendenzählen verdeutlicht: Die Psychologie ist heute nicht nur eines der beliebtesten Studienfächer, sie hat in den letzten Jahren auch ein außerordentliches Wachstum vollzogen. Niemals zuvor studierten mehr Menschen Psychologie in Deutschland als heute (Statistisches Bundesamt, 2019). Wie Abbildung 1 verdeutlicht, verlief die Entwicklung der Studierendenzahlen der letzten 70 Jahre an den (west)deutschen Universitäten und Hochschulen in unterschiedlichen Phasen. Episoden der relativen Stagnation - in den 1980er Jahren, Mitte der 1990er bis 2001 und ein kurzes Intermezzo mit einem Rückgang um rund zehn Prozent zwischen 2001 bis 2007 - wechselten sich ab mit Expansionsschüben steigender Intensität. Auffällig ist der massive Zuwachs seit 2007: Innerhalb von nur zwölf Jahren verdreifachte sich die Zahl der Psychologiestudierenden in Deutschland auf über 90.000! Das entscheidende Momentum für den jüngsten Wachstumsschub geht auf den stark steigenden Anteil weiblicher Studierender zurück. Erstmals überholten sie ihre männlichen Kommilitonen Ende der 1970er Jahre. Seither vergrößerte sich der Abstand zwischen den Geschlechtern zunehmend: 2019 waren mehr als zwei Drittel aller Psychologiestudierenden Frauen. Die Anzahl der männlichen Studierenden stagnierte hingegen ab Anfang der 1980er Jahre und begann erst ab 2010 wieder langsam zu steigen.

Alle Beiträge dieses Themenheftes sind vor dem Hintergrund dieses fachlichen Wachstums zu verstehen. Der Anstieg an Studierenden fällt historisch mit einer Ausdifferenzierung und Expansion psychologischer Berufsfelder zusammen, unter denen das Feld der Psychotherapie eine 


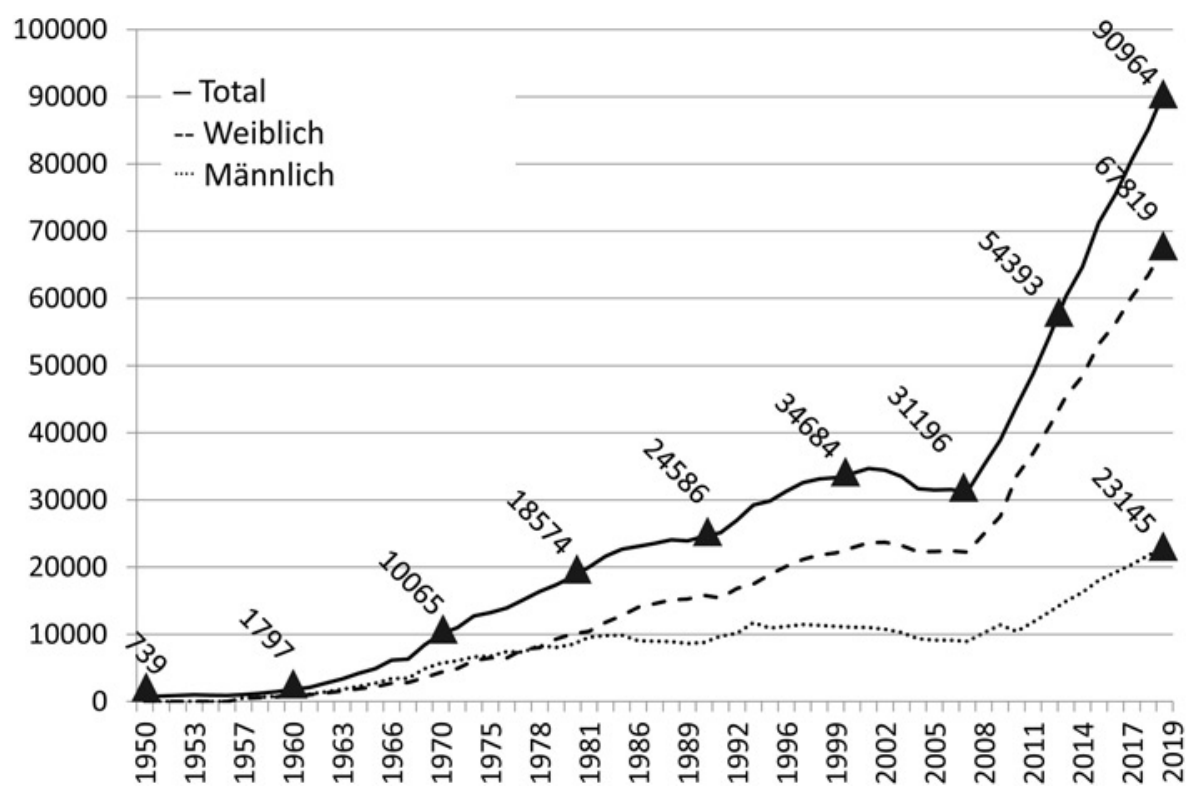

Abbildung 1. Psychologiestudierende an (west)deutschen Universitäten und Hochschulen 1950-2019. Daten aus Hoyos (1964) und Jahrbuch des Statistischen Bundesamts (1959-2020)
Sonderstellung einnimmt. War sie bis zum Zweiten Weltkrieg in Deutschland noch fast ausschließlich in ärztlicher Hand, stieg der Anteil der nichtärztlichen Psychotherapeut_innen in den folgenden Jahrzehnten immer weiter an. 2019 meldete die Kassenärztliche Bundesvereinigung 28.116 Psychologische Psychotherapeut_innen mit Kassensitz, denen 6.219 ärztliche Psychotherapeut_innen gegenüber standen. Die Anziehungskraft dieses Berufsfeldes ist bei den weiblichen Psychologiestudierenden besonders ausgeprägt: Mehr als drei Viertel der nichtärztlichen Psychotherapeut_innen sind heute Frauen (Kassenärztliche Bundesvereinigung, 2019).

In nur zwei Generationen hat sich die deutsche akademische Psychologie von einer Grundlagenwissenschaft, die von einem überschaubaren, männlich dominierten Kreis aus dem Bildungsbürgertum bespielt wurde, in eine stark auf das klinische Feld ausgerichtete, frauendominierte Profession mit bald hunderttausend von Studierenden gewandelt. Viele von ihnen kommen mit bereits relativ klar konturierten Berufswünschen an die Universität. Als Präsident der DGPs berichtete Martin Irle schon Ende der 1970er Jahre von „dominant auf Klinische Psychologie ausgerichteten Berufsvorstellungen der Studierenden" (Irle \& Strack, 1979, S. 1). Das Fach passte sich an, Lehrangebote und Schwerpunktstudiengänge wurden umgestellt. Wenige Jahre später war bei Hörmann und Nestmann von einer aus den USA importierten „Therapeutisierung der Psychologie" zu lesen, welche ihrerseits zur „studentische[n] Präferenz für Klinische Psychologie“ beitrage (Hörmann \& Nestmann, 1985, S. 257).

Mit dem Inkrafttreten des ersten Psychotherapeutengesetzes in Deutschland 1999 wurde der Zugang zur Approbation für Absolvent_innen der Psychologie erstmals bundesweit geregelt, doch begrenzten die Zulassungsbeschränkungen das Wachstum der Studierendenzahlen an den staatlichen Universitäten. Es folgten Ausweichbewegungen ins Ausland, vor allem nach Österreich. Hier stieg der Anteil deutscher Studierender im Fach Psychologie zwischen 2003 und 2018 von $2 \%$ auf $47 \%$ (Kovacs \& Batinic, 2020). Die letzte große Wachstumsphase seit 2007 fällt mit der Umsetzung der Bologna-Reform und der Gründung zahlreicher privater Hochschulen und Universitäten zusammen, die an denen 2020 mit 33.289 Studierenden schon über ein Drittel aller Psychologiestudierenden in Deutschland ausgebildet wurden (Statistisches Bundesamt, 2021).

Die im September 2020 in Kraft getretene Reform der Approbationsordnung lässt sich als Fortsetzung und Konsequenz dieser Entwicklung interpretieren. Durch die umfangreiche Vorverlegung klinischer und praktischer Anteile der Psychotherapieausbildung an die Universität wurden auch neue Reglementarien für Zugangsbeschränkungen zur Psychotherapie geschaffen. Die an den öffentlichen Universitäten angekündigten zweieinhalb bis dreitausend Masterstudienplätze mit Schwerpunkt Klinischer Psychologie und Psychotherapie unterschreiten die Nachfrage auf Seiten der Studierenden bei weitem. Die langfristigen Folgen dieser Entwicklung für den Lehr- und Forschungsbetrieb an den öffentlichen und privaten Hochschulen und Universitäten lassen sich heute nur in Konturen ablesen. Vieles spricht dafür, dass die sich ankündigenden Veränderungen mehr als nur oberflächlich sein werden - Grund genug, um den historischen Tiefenschichten nachzuspüren, auf deren Boden sich diese die jüngste Entwicklung vollzogen hat. 


\section{Die Beiträge des Themenheftes}

Um die jüngsten Transformationsprozesse enger in den Blick zu nehmen, konzentrieren sich die Beiträge des Themenheftes auf die Entwicklungen des psychologischpsychotherapeutischen Feldes in (West-)Deutschland nach dem Zweiten Weltkrieg. Dabei stehen verschiedene inhaltliche Ausrichtungen, bedeutende historische Einschnitte, zentrale institutionelle Etappen und Professionalisierungsmomente im Vordergrund - ebenso wie unterschiedliche psychotherapeutische Ansätze und das Verhältnis zu benachbarten Disziplinen, insbesondere zur Medizin. Aus allen Beiträgen des vorliegenden Heftes scheint hervor, dass die Bruchlinien nicht immer entlang der bekannten disziplinären Grenzen verlaufen, sondern häufig auch quer zu diesen. Weder die Psychologie noch die Medizin bildeten im diskutierten Zeitraum auf wissenschaftstheoretischer oder berufspraktischer Ebene in sich hermetisch geschlossene Einheiten, sondern vereinten unter ihrem Dach verschiedene Schulen und Strömungen, Berufs- und Interessensgruppen.

Der Beitrag von Martin Wieser thematisiert das Verhältnis von akademischer Psychologie und Psychotherapie zur Zeit des Nationalsozialismus. Im Kontext des 1936 gegründeten „Deutschen Instituts für psychologische Forschung und Psychotherapie“ wird die Etablierung des „behandelnden Psychologen“ und der „Deutschen Seelenheilkunde" rekonstruiert und die Bedeutung der berufsrechtlichen Aufwertung der nichtärztlichen Psychotherapeut_innen während des Krieges hervorgehoben.

Lisa Malich nimmt das Verhältnis von Psychologie, Medizin und Psychotherapie in den 1960er und 1970er Jahren in Westdeutschland in den Blick. Schauplatz der mikrohistorischen Untersuchung ist die von Johannes Brengelmann geleitete Psychologische Abteilung des MaxPlanck-Instituts für Psychiatrie, welches bis heute die Rolle eines Geburtsorts im Narrativ von der Verhaltenstherapie als angeblich genuin psychologischer Methode bildet. Diese These stellt die Autorin auf Grundlage von archivarischen Dokumenten in Frage. An dessen Stelle verortet sie ein komplexes und auch widersprüchliches, zwischen Kooperation und Konkurrenz changierendes Verhältnis von Psychologie und Medizin.

Die wiederholten Anläufe zur gesetzlichen Reglementierung der psychotherapeutischen Ausbildung seit den 1960er Jahren rekonstruiert Dietmar Schulte. Im Gegensatz zu den vorangehenden Beiträgen berichtet der Autor auch aus der Sicht eines Zeitzeugen und Akteurs von den bis Ende der 1990er Jahre erfolgten Anläufen um eine Gesetzesreform in der Psychotherapieausbildung. Sozialund berufsrechtliche, gesundheits- und wirtschaftspolitische Kalküle erweisen sich aus dieser Perspektive als (mit) entscheidende Faktoren für die Durchsetzung standespolitischer Interessen.

Den Reigen schließt der Beitrag von Wolfgang Schönpflug, welcher die jüngste Etappe dieses Entwicklungsprozesses in den Blick nimmt. Die vom Autor rekonstruierte Vorgeschichte der 2020 in Kraft getretenen Reform der Psychotherapieausbildung verdeutlicht, dass verschiedene Studienmodelle zur Debatte standen, die auch nach dem Erlass der Gesetzesreform nicht vom Tisch sind: So lässt die jüngste Reform beispielsweise offen, an welchen Fakultäten das neue Studium angeboten werden darf, oder wie die im Gesetz genannten „Bezugswissenschaften" inhaltlich definiert werden.

Abschließend sei noch eine Bemerkung in eigener Sache gestattet: Auch die Geschichte der Psychologie und Psychotherapie ist in der neuen Gesetzesordnung explizit als abzudeckendes Wissensgebiet angeführt. Nicht ohne Grund: Psychologiegeschichte ist Teil psychologischer Grundlagenforschung, sie schafft fachliche Identität und ein kritisches Bewusstsein für die historischen und gesellschaftlichen Ermöglichungsbedingungen psychologischer Forschung und Praxis. Als Sprecherin und Beisitzer der Fachgruppe Geschichte der Psychologie möchten wir unserer Hoffnung Ausdruck verleihen, dass in der Ausbildung der kommenden Studierendengenerationen auch Raum und Zeit hierfür geschaffen wird.

\section{Literatur}

Hörmann, G. \& Nestmann, F. (1985). Die Professionalisierung der Klinischen Psychologie und die Entwicklung neuer Berufsfelder in Beratung, Sozialarbeit und Therapie. In M. Ash \& U. Geuter (Hrsg.), Geschichte der deutschen Psychologie im 20. Jahrhundert (S. 252 - 285). Opladen: Westdeutscher Verlag.

Hoyos, C. G. (1964). Denkschrift zur Lage der Psychologie. Wiesbaden: Steiner.

Irle, M. \& Strack, F. (1979). Psychologie in Deutschland. Ein Bericht zur Lage von Forschung und Lehre. Bonn: Deutsche Forschungsgemeinschaft.

Kassenärztliche Bundesvereinigung. (2019). Statistische Informationen aus dem Bundesarztregister. Verfügbar unter: https:// www.kbv.de/media/sp/2019-12-31_BAR_Statistik.pdf

Kovacs, C. \& Batinic, B. (2020). Psychologie in Österreich. Studiums- und Berufsstatistiken. Psychologische Rundschau, 71, $353-360$.

Rief, W. (2020). Die Zukunft der Psychotherapie nach der Ausbildungsreform. Verhaltenstherapie, 3 (2), 101-103.

Schönpflug, W. (2019). Ist das Ende der Psychologie gekommen? Frankfurter Allgemeine Zeitung, 28.3.2019. Verfügbar unter: https://www.faz.net/aktuell/karriere-hochschule/neues-gesetzist-das-ende-der-psychologie-gekommen-16099105.html

Statistisches Bundesamt. (1959-2020). Statistisches Jahrbuch. Verfügbar unter https://www.destatis.de/DE/Themen/Quer schnitt/Jahrbuch/_inhalt.html

Statistisches Bundesamt. (Destatis). (2019). Bildung und Kultur: Studierende an Hochschulen. Verfügbar unter: https://www.de 
statis.de/DE/Themen/Gesellschaft-Umwelt/Bildung-For schung-Kultur/Hochschulen/Publikationen/Downloads-Hoch schulen/studierende-hochschulen-endg-2110410197004.pdf? _-_blob=publicationFile

Statistisches Bundesamt. (Destatis). (2021). Bildung und Kultur: Private Hochschulen 2019. Verfügbar unter https://www.desta tis.de/DE/Themen/Gesellschaft-Umwelt/Bildung-ForschungKultur/Hochschulen/Publikationen/Downloads-Hochschulen/ private-hochschulen-5213105197004.pdf?__blob=publication File
Tuschen-Caffier, B., Antoni, C., Elsner, B., Bermeitinger, C., Bühner, M., Erdfelder, E. et al. (2020). Quo vadis „Studium und Lehre" in der Psychologie? Denkanstöße für die Neukonzeption von Studiengängen. Psychologische Rundschau, 71, 384-393.

\section{Ass.-Prof. Dr. Dr. Martin Wieser}

Sigmund Freud PrivatUniversität Berlin

Columbiadamm 10, Turm 9

12101 Berlin - Tempelhof

martin.wieser@sfu-berlin.de 